\title{
Exponential Stability of Periodic Solution to Wilson-Cowan Networks with Time-Varying Delays on Time Scales
}

\author{
Jinxiang Cai, Zhenkun Huang, and Honghua Bin \\ School of Science, Jimei University, Xiamen 361021, China \\ Correspondence should be addressed to Zhenkun Huang; hzk974226@jmu.edu.cn
}

Received 31 December 2013; Accepted 12 February 2014; Published 2 April 2014

Academic Editor: Songcan Chen

Copyright (C) 2014 Jinxiang Cai et al. This is an open access article distributed under the Creative Commons Attribution License, which permits unrestricted use, distribution, and reproduction in any medium, provided the original work is properly cited.

\begin{abstract}
We present stability analysis of delayed Wilson-Cowan networks on time scales. By applying the theory of calculus on time scales, the contraction mapping principle, and Lyapunov functional, new sufficient conditions are obtained to ensure the existence and exponential stability of periodic solution to the considered system. The obtained results are general and can be applied to discretetime or continuous-time Wilson-Cowan networks.
\end{abstract}

\section{Introduction}

The activity of a cortical column may be mathematically described through the model developed by Wilson and Cowan $[1,2]$. Such a model consists of two nonlinear ordinary differential equations representing the interactions between two populations of neurons that are distinguished by the fact that their synapses are either excitatory or inhibitory [2]. A comprehensive paper has been done by Destexhe and Sejnowski [3] which summarized all important development and theoretical results for Wilson-Cowan networks. Its extensive applications include pattern analysis and image processing [4]. Theoretical results about the existence of asymptotic stable limit cycle and chaos have been reported in $[5,6]$. Exponential stability of a unique almost periodic solution for delayed Wilson-Cowan type model has been reported in [7]. However, few investigations are fixed on the periodicity of Wilson-Cowan model [8] and it is troublesome to study the stability and periodicity for continuous and discrete system with oscillatory coefficients, respectively. Therefore, it is significant to study Wilson-Cowan networks on time scales $[9,10]$ which can unify the continuous and discrete situations.
Motivated by recent results [11-13], we consider the following dynamic Wilson-Cowan networks on time scale $\mathbb{T}$ :

$$
\begin{aligned}
X_{P}^{\Delta}(t)= & -a_{P}(t) X_{P}(t)+\left[k_{P}(t)-r_{P}(t) X_{P}(t)\right] \\
& \times G\left[w_{P}^{1}(t) X_{P}\left(t-\tau_{P}(t)\right)\right. \\
& \left.-w_{N}^{1}(t) X_{N}\left(t-\tau_{N}(t)\right)+I_{P}(t)\right], \\
X_{N}^{\Delta}(t)= & -a_{N}(t) X_{N}(t)+\left[k_{N}(t)-r_{N}(t) X_{N}(t)\right] \\
& \times G\left[w_{P}^{2}(t) X_{P}\left(t-\tau_{P}(t)\right)\right. \\
& \left.-w_{N}^{2}(t) X_{N}\left(t-\tau_{N}(t)\right)+I_{N}(t)\right],
\end{aligned}
$$

$t \in \mathbb{T}$, where $X_{P}(t), X_{N}(t)$ represent the proportion of excitatory and inhibitory neurons firing per unit time at the instant $t$, respectively. $a_{P}(t)>0$ and $a_{N}(t)>0$ represent the function of the excitatory and inhibitory neurons with natural decay over time, respectively. $r_{P}(t)$ and $r_{N}(t)$ are related to the duration of the refractory period; $k_{P}(t)$ and $k_{N}(t)$ are positive scaling coefficients. $w_{P}^{1}(t), w_{N}^{1}(t), w_{P}^{2}(t)$, and $w_{N}^{2}(t)$ are the strengths of connections between the populations. $I_{P}(t), I_{N}(t)$ are the external inputs to the excitatory and 
the inhibitory populations. $G(\cdot)$ is the response function of neuronal activity. $\tau_{P}(t), \tau_{N}(t)$ correspond to the transmission time-varying delays.

The main aim of this paper is to unify the discrete and continuous Wilson-Cowan networks with periodic coefficients and time-varying delays under one common framework and to obtain some generalized results to ensure the existence and exponential stability of periodic solution on time scales. The main technique is based on the theory of time scales, the contraction mapping principle, and the Lyapunov functional method.

\section{Preliminaries}

In this section, we give some definitions and lemmas on time scales which can be found in books $[14,15]$.

Definition 1. A time scale $\mathbb{T}$ is an arbitrary nonempty closed subset of the real set $\mathbb{R}$. The forward and backward jump operators $\sigma, \rho: \mathbb{T} \rightarrow \mathbb{T}$ and the graininess $\mu: \mathbb{T} \rightarrow \mathbb{R}^{+}$ are defined, respectively, by

$$
\begin{gathered}
\sigma(t):=\inf \{s \in \mathbb{T}: s>t\}, \quad \rho(t):=\sup \{s \in \mathbb{T}: s<t\}, \\
\mu(t):=\sigma(t)-t .
\end{gathered}
$$

These jump operators enable us to classify the point $\{t\}$ of a time scale as right-dense, right-scattered, left-dense, or left-scattered depending on whether

$$
\begin{array}{r}
\sigma(t)=t, \quad \sigma(t)>t, \quad \rho(t)=t, \quad \rho(t)<t, \\
\text { respectively, for any } t \in \mathbb{T} .
\end{array}
$$

The notation $[a, b]_{\mathbb{T}}$ means that $[a, b]_{\mathbb{T}}:=\{t \in \mathbb{T}: a \leq t \leq b\}$. Denote $\mathbb{T}:=\{t \in \mathbb{T}: t \geq 0\}$.

Definition 2. One can say that a time scale $\mathbb{T}$ is periodic if there exists $p>0$ such that $t \in \mathbb{T}$; then $t \pm p \in \mathbb{T}$; the smallest positive number $p$ is called the period of the time scale.

Clearly, if $\mathbb{T}$ is a $p$-periodic time scale, then $\sigma(t+n p)=$ $\sigma(t)+n p$ and $\mu(t+n p)=\mu(t)$. So, $\mu(t)$ is a $p$-periodic function.

Definition 3. Let $\mathbb{T}(\neq \mathbb{R})$ be a periodic time scale with period $p$. One can say that the function $f: \mathbb{T} \rightarrow \mathbb{R}$ is periodic with period $\omega>0$ if there exists a natural number $n$ such that $\omega=n p, f(t+\omega)=f(t)$ for all $t \in \mathbb{T}$ and $\omega$ is the smallest number such that $f(t+\omega)=f(t)$. If $\mathbb{T}=\mathbb{R}$, one can say that $f$ is periodic with period $\omega>0$ if $\omega$ is the smallest positive number such that $f(t+\omega)=f(t)$ for all $t \in \mathbb{R}$.

Definition 4 (Lakshmikantham and Vatsala [16]). For each $t \in \mathbb{T}$, let $N$ be a neighborhood of $t$. Then, one defines the generalized derivative (or Dini derivative), $D^{+} u^{\Delta}(t)$, to mean that, given $\varepsilon>0$, there exists a right neighborhood $N(\varepsilon) \subset N$ of $t$ such that

$$
\frac{u(\sigma(t))-u(s)}{u(t, s)}<D^{+} u^{\Delta}(t)+\varepsilon
$$

for each $s \in N(\varepsilon), s>t$, where $\mu(t, s)=\sigma(t)-s$.
In case $t$ is right-scattered and $u(t)$ is continuous at $t$, one gets

$$
D^{+} u^{\Delta}(t)=\frac{u(\sigma(t))-u(t)}{\sigma(t)-t} .
$$

Definition 5. A function $f: \mathbb{T} \rightarrow \mathbb{R}$ is called rightdense continuous provided that it is continuous at rightdense points of $\mathbb{T}$ and the left-side limit exists (finite) at leftdense continuous functions on $\mathbb{T}$. The set of all right-dense continuous functions on $\mathbb{T}$ is defined by $C_{\mathrm{rd}}=C_{\mathrm{rd}}(\mathbb{T}, \mathbb{R})$.

Definition 6. A function $p: \mathbb{T} \rightarrow \mathbb{\mathbb { T }}$ is called a regressive function if and only if $1+p(t) \mu(t) \neq 0$.

The set of all regressive and right-dense continuous functions is denoted by $\mathscr{R}$. Let $\mathscr{R}^{+}:=\left\{p \in C_{\text {rd }}: 1+\right.$ $p(t) \mu(t)>0$ for all $t \in \mathbb{T}\}$. Next, we give the definition of the exponential function and list its useful properties.

Definition 7 (Bohner and Peterson [14]). If $p \in C_{\mathrm{rd}}$ is a regressive function, then the generalized exponential function $e_{p}(t, s)$ is defined by

$$
e_{p}(t, s)=\exp \left\{\int_{s}^{t} \xi_{\mu(\tau)}(p(\tau)) \Delta \tau\right\}, \quad s, t \in \mathbb{T},
$$

with the cylinder transformation

$$
\xi_{h}(z)= \begin{cases}\frac{\log (1+h z)}{h}, & h \neq 0, \\ z, & h=0 .\end{cases}
$$

Definition 8. The periodic solution

$$
Z^{*}(t)=\left(X_{P}^{*}(t), X_{N}^{*}(t)\right)^{\top}
$$

of (1) is said to be globally exponentially stable if there exists a positive constant $\varepsilon$ and $N=N(\varepsilon)>0$ such that all solutions

$$
Z(t)=\left(X_{P}(t), X_{N}(t)\right)^{\top}
$$

of (1) satisfy

$$
\begin{array}{r}
\left|X_{P}(t)-X_{P}^{*}(t)\right|+\left|X_{N}(t)-X_{N}^{*}(t)\right| \\
\leq N(\epsilon) e_{\ominus \epsilon}(t, \alpha)\left(\sup _{s \in\left[-\tau_{0}, 0\right]_{\mathbb{T}}}\left|X_{P}(s)-X_{P}^{*}(s)\right|\right. \\
\\
\left.\quad+\sup _{s \in\left[-\tau_{0}, 0\right]_{\mathbb{T}}}\left|X_{N}(s)-X_{N}^{*}(s)\right|\right), \\
\quad t \in \mathbb{T} .
\end{array}
$$

Lemma 9 (Bohner and Peterson [15]). If $p, q \in \mathscr{R}$, then

(i) $e_{0}(t, s) \equiv 1$ and $e_{p}(t, t) \equiv 1$;

(ii) $e_{p}(\sigma(t), s)=(1+\mu(t) p(t)) e_{p}(t, s)$;

(iii) $1 / e_{p}(t, s)=e_{\ominus p}(t, s)$, where $\ominus p(t)=-p(t) /(1+$ $\mu(t) p(t))$ 

(iv) $e_{p}(t, s)=1 / e_{p}(s, t)=e_{\ominus p}(s, t)$;
(v) $e_{p}(t, s) e_{p}(s, r)=e_{p}(t, r)$;
(vi) $e_{p}(t, s) e_{q}(t, s)=e_{p \oplus q}(t, s)$;
(vii) $e_{p}(t, s) / e_{q}(t, s)=e_{p \ominus q}(t, s)$;
(viii) $\left(1 / e_{p}(\cdot, s)\right)^{\Delta}=-p(t) / e_{p}^{\sigma}(\cdot, s)$.

Lemma 10 (contraction mapping principle [17]). If $\Omega$ is a closed subset of a Banach space $X$ and $\mathscr{F}: \Omega \rightarrow \Omega$ is a contraction, then $\mathscr{F}$ has a unique fixed point in $\Omega$.

For any $\omega$-periodic function $\mathscr{V}$ defined on $\mathbb{T}$, denote $\overline{\mathscr{V}}=$ $\max _{t \in[0, \omega]} \mathscr{V}(t), \underline{\mathscr{V}}=\min _{t \in[0, \omega]} \mathscr{V}(t), \overline{|\mathscr{V}|}=\max _{t \in[0, \omega]}|\mathscr{V}(t)|$, and $|\mathscr{V}|=\min _{t \in[0, \omega]}|\mathscr{V}(t)|$. Throughout this paper, we make the following assumptions:

$\left(A_{1}\right) k_{P}(t), k_{N}(t), r_{P}(t), r_{N}(t), w_{P}^{1}(t), w_{P}^{2}(t), w_{N}^{1}(t), w_{N}^{2}(t)$, $a_{P}(t), a_{N}(t), \tau_{P}(t), \tau_{N}(t), I_{P}(t)$, and $I_{N}(t)$ are $\omega$ periodic functions defined on $\mathbb{T},-a_{P}(t),-a_{N}(t) \in$ $\mathscr{R}^{+}$.

$\left(A_{2}\right) G(\cdot): \mathbb{R} \rightarrow \mathbb{R}$ is Lipschitz continuous; that is, $|G(u)-G(v)| \leq L|u-v|$, for all $u, v \in \mathbb{R}$, and $G(0)=0$, $\sup _{v \in \mathbb{R}}|G(v)| \leq M$.

For simplicity, take the following denotations:

$$
\begin{gathered}
R=\max \left\{\overline{r_{P}}, \overline{r_{N}}\right\}, \quad I=\max \left\{L \overline{\left|I_{P}\right|}, L \mid \overline{I_{N} \mid}\right\}, \\
K=\max \left\{\overline{k_{P}}, \overline{k_{N}}\right\}, \\
W=\max \left\{L \overline{w_{P}^{1}}, L \overline{w_{N}^{1}}, L \overline{w_{P}^{2}}, \overline{L w_{N}^{2}}\right\}, \\
\tau_{0}=\min \left\{\left|\underline{\tau_{P} \mid},\right| \tau_{N} \mid\right\} .
\end{gathered}
$$

Lemma 11. Suppose $\left(A_{1}\right)$ holds; then $Z(t)$ is an $\omega$-periodic solution of (1) if and only if $Z(t)$ is the solution of the following system:

$$
\begin{aligned}
X_{P}(t)= & \frac{1}{e_{\ominus\left(-a_{P}\right)}(\omega, 0)-1} \\
& \times \int_{t}^{t+\omega} \frac{e_{\ominus\left(-a_{P}\right)}(s, t)}{1-\mu(s) a_{P}(s)}\left[k_{P}(s)-r_{P}(s) X_{P}(s)\right] \\
& \times G\left[w_{P}^{1}(s) X_{P}\left(s-\tau_{P}(s)\right)\right. \\
X_{N}(t)= & \frac{\left.-w_{N}^{1}(s) X_{N}\left(s-\tau_{N}(s)\right)+I_{P}(s)\right] \Delta s}{e_{\ominus\left(-a_{N}\right)}(\omega, 0)-1} \\
& \times \int_{t \quad t+\omega}^{t \quad} \frac{e_{\ominus\left(-a_{N}\right)}(s, t)}{1-\mu(s) a_{N}(s)}\left[k_{N}(s)-r_{N}(s) X_{N}(s)\right] \\
\quad & \quad G\left[w_{P}^{2}(s) X_{P}\left(s-\tau_{P}(s)\right)\right. \\
& \left.-w_{N}^{2}(s) X_{N}\left(s-\tau_{N}(s)\right)+I_{N}(s)\right] \Delta s .
\end{aligned}
$$

Proof. Let $Z(t)=\left(X_{P}(t), X_{N}(t)\right)^{\top}$ be a solution of (1); we can rewrite (1) as follows:

$$
\begin{aligned}
& X_{P}^{\Delta}(t)+ a_{P}(t)\left(X_{P}^{\sigma}(t)-\mu(t) X_{P}^{\Delta}(t)\right) \\
&= {\left[k_{P}(t)-r_{P}(t) X_{P}(t)\right] } \\
& \times G\left[w_{P}^{1}(t) X_{P}\left(t-\tau_{P}(t)\right)\right. \\
&\left.-w_{N}^{1}(t) X_{N}\left(t-\tau_{N}(t)\right)+I_{P}(t)\right], \\
& X_{N}^{\Delta}(t)+a_{N}(t)\left(X_{N}^{\sigma}(t)-\mu(t) X_{N}^{\Delta}(t)\right) \\
&=\left[k_{N}(t)-r_{N}(t) X_{N}(t)\right] \\
& \times G\left[w_{P}^{2}(t) X_{P}\left(t-\tau_{P}(t)\right)\right. \\
&\left.\quad-w_{N}^{2}(t) X_{N}\left(t-\tau_{N}(t)\right)+I_{N}(t)\right],
\end{aligned}
$$

which leads to

$$
\begin{aligned}
& X_{P}^{\Delta}(t)+\ominus\left(-a_{P}\right)(t) X_{P}^{\sigma}(t) \\
& =\left[k_{P}(t)-r_{P}(t) X_{P}(t)\right] \\
& \times G\left[w_{P}^{1}(t) X_{P}\left(t-\tau_{P}(t)\right)\right. \\
& \left.\quad-w_{N}^{1}(t) X_{N}\left(t-\tau_{N}(t)\right)+I_{P}(t)\right] \frac{1}{1-\mu(t) a_{P}(t)}, \\
& X_{N}^{\Delta}(t)+\ominus\left(-a_{N}\right)(t) X_{N}^{\sigma}(t) \\
& =\left[k_{N}(t)-r_{N}(t) X_{N}(t)\right] \\
& \times G\left[w_{P}^{2}(t) X_{P}\left(t-\tau_{P}(t)\right)\right. \\
& \left.\quad-w_{N}^{2}(t) X_{N}\left(t-\tau_{N}(t)\right)+I_{N}(t)\right] \frac{1}{1-\mu(t) a_{N}(t)} .
\end{aligned}
$$

Multiplying both sides of the above equalities by $e_{\ominus\left(-a_{P}\right)}(t, 0)$ and $e_{\ominus\left(-a_{N}\right)}(t, 0)$, respectively, we have

$$
\begin{aligned}
& {\left[e_{\ominus\left(-a_{P}\right)}(t, 0) X_{P}(t)\right]^{\Delta}} \\
& =\left[k_{P}(t)-r_{P}(t) X_{P}(t)\right] \\
& \quad \times G\left[w_{P}^{1}(t) X_{P}\left(t-\tau_{P}(t)\right)\right. \\
& \left.\quad-w_{N}^{1}(t) X_{N}\left(t-\tau_{N}(t)\right)+I_{P}(t)\right] e_{\ominus\left(-a_{P}\right)}(\sigma(t), 0) \\
& {\left[e_{\ominus\left(-a_{N}\right)}(t, 0) X_{N}(t)\right]^{\Delta}} \\
& =\left[k_{N}(t)-r_{N}(t) X_{N}(t)\right] \\
& \quad \times G\left[w_{P}^{2}(t) X_{P}\left(t-\tau_{P}(t)\right)\right. \\
& \left.\quad-w_{N}^{2}(t) X_{N}\left(t-\tau_{N}(t)\right)+I_{N}(t)\right] \\
& \quad \times e_{\ominus\left(-a_{N}\right)}(\sigma(t), 0) .
\end{aligned}
$$


Integrating both sides of the above equalities from $t$ to $t+\omega$ and using $X_{P}(t+\omega)=X_{P}(t)$ and $X_{N}(t+\omega)=X_{N}(t)$, we have

$$
\begin{aligned}
& X_{P}(t)=\int_{t}^{t+\omega}\left[\left[k_{P}(s)-r_{P}(s) X_{P}(s)\right]\right. \\
& \times G\left[w_{P}^{1}(s) X_{P}\left(s-\tau_{P}(s)\right)\right. \\
& \left.\left.-w_{N}^{1}(s) X_{N}\left(s-\tau_{N}(s)\right)+I_{P}(s)\right]\right] \\
& \times \frac{e_{\ominus\left(-a_{P}\right)}(\sigma(s), 0)}{e_{\ominus\left(-a_{P}\right)}(t+\omega, 0)-e_{\ominus\left(-a_{P}\right)}(t, 0)} \Delta s \\
& =\int_{t}^{t+\omega}\left[\left[k_{P}(s)-r_{P}(s) X_{P}(s)\right]\right. \\
& \times G\left[w_{P}^{1}(s) X_{P}\left(s-\tau_{P}(s)\right)\right. \\
& \left.\left.-w_{N}^{1}(s) X_{N}\left(s-\tau_{N}(s)\right)+I_{P}(s)\right]\right] \\
& \times \frac{e_{\ominus\left(-a_{P}\right)}(\sigma(s), t)}{e_{\ominus\left(-a_{P}\right)}(t+\omega, t)-1} \Delta s, \\
& X_{N}(t)=\int_{t}^{t+\omega}\left[\left[k_{N}(s)-r_{N}(s) X_{N}(s)\right]\right. \\
& \times G\left[w_{P}^{2}(s) X_{P}\left(s-\tau_{P}(s)\right)\right. \\
& \left.\left.-w_{N}^{2}(s) X_{N}\left(s-\tau_{N}(s)\right)+I_{N}(s)\right]\right] \\
& \times \frac{e_{\ominus\left(-a_{N}\right)}(\sigma(s), 0)}{e_{\ominus\left(-a_{N}\right)}(t+\omega, 0)-e_{\ominus\left(-a_{N}\right)}(t, 0)} \Delta s \\
& =\int_{t}^{t+\omega}\left[\left[k_{N}(s)-r_{N}(s) X_{N}(s)\right]\right. \\
& \times G\left[w_{P}^{2}(s) X_{P}\left(s-\tau_{P}(s)\right)\right. \\
& \left.\left.-w_{N}^{2}(s) X_{N}\left(s-\tau_{N}(s)\right)+I_{N}(s)\right]\right] \\
& \times \frac{e_{\ominus\left(-a_{N}\right)}(\sigma(s), t)}{e_{\ominus\left(-a_{N}\right)}(t+\omega, t)-1} \Delta s .
\end{aligned}
$$

Since

$$
\begin{aligned}
& \frac{e_{\ominus\left(-a_{P}\right)}(s, t)}{1-\mu(s) a_{P}(s)}=e_{\ominus\left(-a_{P}\right)}(\sigma(s), t), \\
& \frac{e_{\ominus\left(-a_{N}\right)}(s, t)}{1-\mu(s) a_{N}(s)}=e_{\ominus\left(-a_{N}\right)}(\sigma(s), t)
\end{aligned}
$$

and $a_{P}(t+\omega)=a_{P}(t), a_{N}(t+\omega)=a_{N}(t)$, we obtain that

$$
\begin{aligned}
& X_{P}(t)= \frac{1}{e_{\ominus\left(-a_{P}\right)}(\omega, 0)-1} \\
& \times \int_{t}^{t+\omega} \frac{e_{\ominus\left(-a_{P}\right)}(s, t)}{1-\mu(s) a_{P}(s)}\left[k_{P}(s)-r_{P}(s) X_{P}(s)\right] \\
& \quad \times G\left[w_{P}^{1}(s) X_{P}\left(s-\tau_{P}(s)\right)\right. \\
&\left.\quad-w_{N}^{1}(s) X_{N}\left(s-\tau_{N}(s)\right)+I_{P}(s)\right] \Delta s,
\end{aligned}
$$

$$
\begin{aligned}
& X_{N}(t)= \frac{1}{e_{\ominus\left(-a_{N}\right)}(\omega, 0)-1} \\
& \quad \times \int_{t}^{t+\omega} \frac{e_{\ominus\left(-a_{N}\right)}(s, t)}{1-\mu(s) a_{N}(s)}\left[k_{N}(s)-r_{N}(s) X_{N}(s)\right] \\
& \quad \times G\left[w_{P}^{2}(s) X_{P}\left(s-\tau_{P}(s)\right)\right. \\
&\left.\quad-w_{N}^{2}(s) X_{N}\left(s-\tau_{N}(s)\right)+I_{N}(s)\right] \Delta s .
\end{aligned}
$$

The proof is completed.

\section{Main Results}

In this section, we prove the existence and uniqueness of the periodic solution to (1).

Theorem 12. Suppose $\left(A_{1}\right)-\left(A_{2}\right)$ hold and $\max \{\alpha, W\}<1$. Then (1) has a unique $\omega$-periodic solution, where

$$
\begin{aligned}
& \alpha_{1}:=\frac{\omega \exp \left(\int_{0}^{\omega}\left|\xi_{\mu(\tau)} \ominus\left(-a_{P}(\tau)\right)\right| \Delta \tau\right)(K+R \beta+R M / W)}{\left|e_{\ominus\left(-a_{P}\right)}(\omega, 0)-1\right|\left(1-\overline{a_{P}} \bar{\mu}\right)}, \\
& \alpha_{2}:=\frac{\omega \exp \left(\int_{0}^{\omega}\left|\xi_{\mu(\tau)} \ominus\left(-a_{N}(\tau)\right)\right| \Delta \tau\right)(K+R \beta+R M / W)}{\left|e_{\ominus\left(-a_{N}\right)}(\omega, 0)-1\right|\left(1-\overline{a_{P}} \bar{\mu}\right)},
\end{aligned}
$$

and $\alpha:=\max \left\{\alpha_{1}, \alpha_{2}\right\}$.

Proof. Let $\mathbb{X}=\left\{Z(t)=\left(z_{P}(t), z_{N}(t)\right) \mid Z \in C_{\mathrm{rd}}\left(\mathbb{T}, \mathbb{R}^{2}\right), Z(t+\right.$ $\omega)=Z(t)\}$ with the norm $\|Z\|=\sup _{t \in \mathbb{T}}\left\{\left|z_{P}(t)\right|+\left|z_{N}(t)\right|\right\}$; then $\mathbb{X}$ is a Banach space [14]. Define

$$
\mathscr{F}: \mathbb{X} \longrightarrow \mathbb{X}, \quad(\mathscr{F} Z)(t)=\left((\mathscr{F} Z)_{P}(t),(\mathscr{F} Z)_{N}(t)\right),
$$

where $Z(t)=\left(z_{P}(t), z_{N}(t)\right) \in \mathbb{X}$ and

$$
\begin{aligned}
(\mathscr{F} Z)_{P}(t)= & \frac{1}{e_{\ominus\left(-a_{P}\right)}(\omega, 0)-1} \\
& \times \int_{t}^{t+\omega} \frac{e_{\ominus\left(-a_{P}\right)}(s, t)}{1-\mu(s) a_{P}(s)}\left[k_{P}(s)-r_{P}(s) z_{P}(s)\right] \\
& \times G\left[w_{P}^{1}(s) z_{P}\left(s-\tau_{P}(s)\right)\right. \\
& \left.\quad-w_{N}^{1}(s) z_{N}\left(s-\tau_{N}(s)\right)+I_{P}(s)\right] \Delta s,
\end{aligned}
$$

$(\mathscr{F} Z)_{N}(t)=\frac{1}{e_{\ominus\left(-a_{N}\right)}(\omega, 0)-1}$

$$
\begin{aligned}
\times \int_{t}^{t+\omega} \frac{e_{\ominus\left(-a_{N}\right)}(s, t)}{1-\mu(s) a_{N}(s)}\left[k_{N}(s)-r_{N}(s) z_{N}(s)\right] \\
\times G\left[w_{P}^{2}(s) z_{P}\left(s-\tau_{P}(s)\right)\right. \\
\left.\quad-w_{N}^{2}(s) z_{N}\left(s-\tau_{N}(s)\right)+I_{N}(s)\right] \Delta s
\end{aligned}
$$


for $t \in \mathbb{T}$. Note that

$$
\begin{aligned}
e_{\ominus\left(-a_{P}\right)}(s, t) & =e^{\int_{t}^{s} \xi_{\mu(\tau)}\left(\Theta\left(-a_{P}\right)(\tau)\right) \Delta \tau} \\
& \leq e^{\int_{t}^{t+\omega}\left|\xi_{\mu(\tau)}\left(\Theta\left(-a_{P}\right)(\tau)\right)\right| \Delta \tau} \\
& =e^{\int_{0}^{\omega}\left|\xi_{\mu(\tau)}\left(\Theta\left(-a_{P}\right)(\tau)\right)\right| \Delta \tau} .
\end{aligned}
$$

Let $\Omega=\{Z(t) \mid Z \in \mathbb{X},\|Z\| \leq I /(1-W)\}$ and $\beta:=I /(1-W)$. Obviously, $\Omega$ is a closed nonempty subset of $\mathbb{X}$. Firstly, we prove that the mapping $\mathscr{F}$ maps $\Omega$ into itself. In fact, for any $Z(t) \in \Omega$, we have

$$
\begin{aligned}
\left|(\mathscr{F} Z)_{P}(t)\right| & \mid \frac{1}{e_{\ominus\left(-a_{P}\right)}(\omega, 0)-1} \\
& \times \int_{t}^{t+\omega} \frac{e_{\ominus\left(-a_{P}\right)}(s, t)}{1-\mu(s) a_{P}(s)}\left[k_{P}(s)-r_{P}(s) z_{P}(s)\right] \\
& \times G\left[w_{P}^{1}(s) z_{P}\left(s-\tau_{P}(s)\right)\right. \\
\leq & \frac{\exp \left(\int_{0}^{\omega}\left|\xi_{\mu(\tau)}\left(\ominus\left(-a_{P}\right)(\tau)\right)\right| \Delta \tau\right)}{\left|e_{\ominus\left(-a_{P}\right)}(\omega, 0)-1\right|\left(1-\overline{a_{P}} \bar{\mu}\right)} \\
& \times \int_{t}^{t+\omega} \mid\left[k_{P}(s)-r_{P}(s) z_{P}(s)\right] \\
\leq & \alpha_{1}\left(I+W \sup _{N}\left(\left|z_{P}(t)\right|+\left|z_{N}(t)\right|\right)\right) \\
\leq & \left.\quad \times I_{t}(s)\right] \Delta s \mid \\
& \left.\quad-w_{N}^{1}(s) z_{N}\left(s-\tau_{N}(s)\right)+I_{P}(s)\right] \Delta s \mid \\
& \exp \left(\int_{0}^{\omega}\left|\xi_{\mu(\tau)}\left(\ominus\left(-a_{P}\right)(\tau)\right)\right| \Delta \tau\right)(K+R \beta)
\end{aligned}
$$

Similarly, we have

$$
\begin{aligned}
& \left|(\mathscr{F} Z)_{N}(t)\right| \\
& =\mid \frac{1}{e_{\ominus\left(-a_{N}\right)}(\omega, 0)-1} \\
& \quad \times \int_{t}^{t+\omega} \frac{e_{\ominus\left(-a_{N}\right)}(s, t)}{1-\mu(s) a_{N}(s)}\left[k_{N}(s)-r_{N}(s) z_{P}(s)\right] \\
& \quad \times G\left[w_{P}^{2}(s) z_{N}\left(s-\tau_{P}(s)\right)\right. \\
& \left.\quad-w_{N}^{2}(s) z_{N}\left(s-\tau_{N}(s)\right)+I_{N}(s)\right] \Delta s \mid \\
& \leq \alpha_{2}\left(I+W \sup _{t \in \mathbb{T}}\left(\left|z_{P}(t)\right|+\left|z_{N}(t)\right|\right)\right) .
\end{aligned}
$$

It follows from (23) and (24) that

$$
\|\mathscr{F} Z\| \leq \alpha I+\alpha W\|Z\| \leq \frac{I}{1-W} .
$$

Hence, $\mathscr{F} Z \in \Omega$.

Next, we prove that $\mathscr{F}$ is a contraction mapping. For any $Z(t)=\left(z_{P}(t), z_{N}(t)\right) \in \Omega, Z^{\prime}(t)=\left(z_{P}^{\prime}(t), z_{N}^{\prime}(t)\right) \in \Omega$, we have

$$
\begin{aligned}
& \left|(\mathscr{F} Z)_{P}(t)-\left(\mathscr{F} Z^{\prime}\right)_{P}(t)\right| \\
& =\mid \frac{1}{e_{\ominus\left(-a_{P}\right)}(\omega, 0)-1} \\
& \times \int_{t}^{t+\omega} \frac{e_{\ominus\left(-a_{P}\right)}(s, t)}{1-\mu(s) a_{P}(s)}\left[k_{P}(s)-r_{P}(s) z_{P}(s)\right] \\
& \times G\left[w_{P}^{1}(s) z_{P}\left(s-\tau_{P}(s)\right)\right. \\
& \left.-w_{N}^{1}(s) z_{N}\left(s-\tau_{N}(s)\right)+I_{P}(s)\right] \Delta s \\
& -\frac{1}{e_{\ominus\left(-a_{P}\right)}(\omega, 0)-1} \\
& \times \int_{t}^{t+\omega} \frac{e_{\ominus\left(-a_{P}\right)}(s, t)}{1-\mu(s) a_{P}(s)}\left[k_{P}(s)-r_{P}(s) z_{P}^{\prime}(s)\right] \\
& \times G\left[w_{P}^{1}(s) z_{P}^{\prime}\left(s-\tau_{P}(s)\right)\right. \\
& \left.-w_{N}^{1}(s) z_{N}^{\prime}\left(s-\tau_{N}(s)\right)+I_{P}(s)\right] \Delta s \\
& \leq \frac{\exp \left(\int_{0}^{\omega}\left|\xi_{\mu(\tau)}\left(\ominus\left(-a_{P}\right)(\tau)\right)\right| \Delta \tau\right)(K W+R W \beta+R M)}{\left|e_{\ominus\left(-a_{P}\right)}(\omega, 0)-1\right|\left(1-\overline{a_{P} \mu}\right)} \\
& \times \int_{t}^{t+\omega} \mid z_{P}\left(s-\tau_{P}(s)\right)-z_{P}^{\prime}\left(s-\tau_{P}(s)\right)+z_{N}\left(s-\tau_{N}(s)\right) \\
& -z_{N}^{\prime}\left(s-\tau_{N}(s)\right) \mid \Delta s \\
& \leq \alpha_{1} W \sup _{t \in \mathbb{T}}\left[\left|z_{P}(t)-z_{P}^{\prime}(t)\right|+\left|z_{N}(t)-z_{N}^{\prime}(t)\right|\right] .
\end{aligned}
$$

Similarly, we have

$$
\begin{aligned}
& \left|(\mathscr{F} Z)_{N}(t)-\left(\mathscr{F} Z^{\prime}\right)_{N}(t)\right| \\
& =\mid \frac{1}{e_{\ominus\left(-a_{N}\right)}(\omega, 0)-1} \\
& \quad \times \int_{t}^{t+\omega} \frac{e_{\ominus\left(-a_{N}\right)}(s, t)}{1-\mu(s) a_{N}(s)}\left[k_{N}(s)-r_{N}(s) z_{N}(s)\right] \\
& \quad \times G\left[w_{P}^{2}(s) z_{P}\left(s-\tau_{P}(s)\right)\right. \\
& \left.\quad-w_{N}^{2}(s) z_{N}\left(s-\tau_{N}(s)\right)+I_{N}(s)\right] \Delta s
\end{aligned}
$$




$$
\begin{gathered}
-\frac{1}{e_{\ominus\left(-a_{N}\right)}(\omega, 0)-1} \\
\times \int_{t}^{t+\omega} \frac{e_{\ominus\left(-a_{N}\right)}(s, t)}{1-\mu(s) a_{N}(s)}\left[k_{N}(s)-r_{N}(s) z_{N}^{\prime}(s)\right] \\
\times G\left[w_{P}^{2}(s) z_{P}^{\prime}\left(s-\tau_{P}(s)\right)\right. \\
\left.-w_{N}^{2}(s) z_{N}^{\prime}\left(s-\tau_{N}(s)\right)+I_{N}(s)\right] \Delta s \mid \\
\leq \alpha_{2} W \sup _{t \in \mathbb{T}}\left[\left|z_{P}(t)-z_{P}^{\prime}(t)\right|+\left|z_{N}(t)-z_{N}^{\prime}(t)\right|\right] .
\end{gathered}
$$

From (26) and (27), we can get

$$
\left\|(\mathscr{F} Z)-\left(\mathscr{F} Z^{\prime}\right)\right\| \leq \alpha W\left\|Z-Z^{\prime}\right\|
$$

Note that $\alpha W<1$. Thus, $\mathscr{F}$ is a contraction mapping. By the fixed point theorem in the Banach space, $\mathscr{F}$ possesses a unique fixed point. The proof is completed.

Theorem 13. Under the conditions of Theorem 12, suppose further the following.

$\left(A_{3}\right)$ There exist some constants $\epsilon>0, \xi>0, \xi^{\prime}>0$ such that

$$
\begin{aligned}
& \left(1+\frac{\xi^{\prime}}{\xi}\right) \frac{\left(1+\epsilon \mu\left(t+\tau_{0}\right)\right)(K+R \beta) W}{\left(\underline{a_{P}}-R M\right)(1+\epsilon \mu(t))-\epsilon} e_{\epsilon}\left(t+\tau_{0}, t\right)<1, \\
& \left(1+\frac{\xi}{\xi^{\prime}}\right) \frac{\left(1+\epsilon \mu\left(t+\tau_{0}\right)\right)(K+R \beta) W}{\left(\underline{a_{N}}-R M\right)(1+\epsilon \mu(t))-\epsilon} e_{\epsilon}\left(t+\tau_{0}, t\right)<1
\end{aligned}
$$

then the periodic solution of (1) is globally exponentially stable.

Proof. It follows from Theorem 12 that (1) has an $\omega$-periodic solution $Z^{*}=\left(X_{P}^{*}(t), X_{N}^{*}(t)\right)^{\top}$. have

Let $Z(t)=\left(X_{P}(t), X_{N}(t)\right)^{\top}$ be any solution of (1); then we

$$
\begin{aligned}
& \left(X_{P}(t)-X_{P}^{*}(t)\right)^{\Delta} \\
& =-a_{P}(t)\left(X_{P}(t)-X_{P}^{*}(t)\right) \\
& +k_{P}(t) G\left[w_{P}^{1}(t) X_{P}\left(t-\tau_{P}(t)\right)\right. \\
& \left.-w_{N}^{1}(t) X_{N}\left(t-\tau_{N}(t)\right)+I_{P}(t)\right] \\
& -k_{P}(t) G\left[w_{P}^{1}(t) X_{P}^{*}\left(t-\tau_{P}(t)\right)\right. \\
& \left.-w_{N}^{1}(t) X_{N}^{*}\left(t-\tau_{N}(t)\right)+I_{P}(t)\right] \\
& -r_{P}(t) X_{P}(t) G\left[w_{P}^{1}(t) X_{P}\left(t-\tau_{P}(t)\right)\right. \\
& \left.-w_{N}^{1}(t) X_{N}\left(t-\tau_{N}(t)\right)+I_{P}(t)\right] \\
& +r_{P}(t) X_{P}^{*}(t) G\left[w_{P}^{1}(t) X_{P}^{*}\left(t-\tau_{P}(t)\right)\right. \\
& \left.-w_{N}^{1}(t) X_{N}^{*}\left(t-\tau_{N}(t)\right)+I_{P}(t)\right]
\end{aligned}
$$

$$
\begin{gathered}
\left(X_{N}(t)-X_{N}^{*}(t)\right)^{\Delta} \\
=-a_{N}(t)\left(X_{N}(t)-X_{N}^{*}(t)\right) \\
+k_{N}(t) G\left[w_{P}^{2}(t) X_{P}\left(t-\tau_{P}(t)\right)\right. \\
\left.-w_{N}^{2}(t) X_{N}\left(t-\tau_{N}(t)\right)+I_{N}(t)\right] \\
-k_{N}(t) G\left[w_{P}^{2}(t) X_{P}^{*}\left(t-\tau_{P}(t)\right)\right. \\
\left.-w_{N}^{2}(t) X_{N}^{*}\left(t-\tau_{N}(t)\right)+I_{N}(t)\right] \\
-r_{N}(t) X_{N}(t) G\left[w_{P}^{2}(t) X_{P}\left(t-\tau_{P}(t)\right)\right. \\
\left.-w_{N}^{2}(t) X_{N}\left(t-\tau_{N}(t)\right)+I_{N}(t)\right] \\
+r_{N}(t) X_{N}^{*}(t) G\left[w_{P}^{2}(t) X_{P}^{*}\left(t-\tau_{P}(t)\right)\right. \\
\left.-w_{N}^{2}(t) X_{N}^{*}\left(t-\tau_{N}(t)\right)+I_{N}(t)\right]
\end{gathered}
$$

which leads to

$$
\begin{aligned}
& D^{+}\left|X_{P}(t)-X_{P}^{*}(t)\right|^{\Delta} \\
& \leq-\left(\underline{a_{P}}-R M\right)\left|X_{P}(t)-X_{P}^{*}(t)\right|+(K+R \beta) W \\
& \times\left(\left|X_{P}\left(t-\tau_{0}\right)-X_{P}^{*}\left(t-\tau_{0}\right)\right|\right. \\
& \left.+\left|X_{N}\left(t-\tau_{0}\right)-X_{N}^{*}\left(t-\tau_{0}\right)\right|\right) \\
& D^{+}\left|X_{N}(t)-X_{N}^{*}(t)\right|^{\Delta} \\
& \leq-\left(\underline{a_{N}}-R M\right)\left|X_{N}(t)-X_{N}^{*}(t)\right|+(K+R \beta) W \\
& \times\left(\left|X_{P}\left(t-\tau_{0}\right)-X_{P}^{*}\left(t-\tau_{0}\right)\right|\right. \\
& \left.+\left|X_{N}\left(t-\tau_{0}\right)-X_{N}^{*}\left(t-\tau_{0}\right)\right|\right) .
\end{aligned}
$$

For any $\alpha \in\left[-\tau_{0}, 0\right]_{\mathbb{T}}$, construct the Lyapunov functional $V(t)=V_{1}(t)+V_{2}(t)+V_{3}(t)+V_{4}(t)$, where

$$
\begin{aligned}
& V_{1}(t)=\xi e_{\epsilon}(t, \alpha)\left|X_{P}(t)-X_{P}^{*}(t)\right|, \\
& V_{3}(t)= \xi^{\prime} e_{\epsilon}(t, \alpha)\left|X_{N}(t)-X_{N}^{*}(t)\right|, \\
& V_{2}(t)=\xi \int_{t-\tau_{0}}^{t}\left(1+\epsilon \mu\left(s+\tau_{0}\right)\right) e_{\epsilon}\left(s+\tau_{0}, \alpha\right)(K+R \beta) W \\
& \quad \times\left(\left|X_{P}(s)-X_{P}^{*}(s)\right|+\left|X_{N}(s)-X_{N}^{*}(s)\right|\right) \Delta s, \\
& V_{4}(t)=\xi^{\prime} \int_{t-\tau_{0}}^{t}\left(1+\epsilon \mu\left(s+\tau_{0}\right)\right) e_{\epsilon}\left(s+\tau_{0}, \alpha\right)(K+R \beta) W \\
& \quad \times\left(\left|X_{P}(s)-X_{P}^{*}(s)\right|+\left|X_{N}(s)-X_{N}^{*}(s)\right|\right) \Delta s .
\end{aligned}
$$


Calculating $D^{+} V(t)^{\Delta}$ along (1), we can get

$$
\begin{aligned}
& \left.D^{+} V_{1}(t)^{\Delta}\right|_{(1)} \\
& \leq \xi\left[\epsilon e_{\epsilon}(t, \alpha)\left|X_{P}(t)-X_{P}^{*}(t)\right|\right. \\
& \left.+e_{\epsilon}(\sigma(t), \alpha) D^{+}\left|X_{P}(t)-X_{P}^{*}(t)\right|^{\Delta}\right] \\
& \leq \xi\left\{\epsilon e_{\epsilon}(t, \alpha)\left|X_{P}(t)-X_{P}^{*}(t)\right|+e_{\epsilon}(\sigma(t), \alpha)\right. \\
& \times\left[-\left(\underline{a_{P}}-R M\right)\left|X_{P}(t)-X_{P}^{*}(t)\right|+(K+R \beta) W\right. \\
& \times\left(\left|X_{P}\left(t-\tau_{0}\right)-X_{P}^{*}\left(t-\tau_{0}\right)\right|\right. \\
& \left.\left.\left.+\left|X_{N}\left(t-\tau_{0}\right)-X_{N}^{*}\left(t-\tau_{0}\right)\right|\right)\right]\right\} \\
& =\xi\left[\epsilon-\left(\underline{a_{P}}-R M\right)(1+\epsilon \mu(t))\right] e_{\epsilon}(t, \alpha)\left|X_{P}(t)-X_{P}^{*}(t)\right| \\
& +\xi(1+\epsilon \mu(t)) e_{\epsilon}(t, \alpha)(K+R \beta) W \\
& \times\left(\left|X_{P}\left(t-\tau_{0}\right)-X_{P}^{*}\left(t-\tau_{0}\right)\right|\right. \\
& \left.+\left|X_{N}\left(t-\tau_{0}\right)-X_{N}^{*}\left(t-\tau_{0}\right)\right|\right), \\
& \left.D^{+} V_{2}(t)^{\Delta}\right|_{(1)} \\
& \leq \xi\left(1+\epsilon \mu\left(t+\tau_{0}\right)\right) e_{\epsilon}\left(t+\tau_{0}, \alpha\right)(K+R \beta) W \\
& \times\left(\left|X_{P}(t)-X_{P}^{*}(t)\right|+\left|X_{N}(t)-X_{N}^{*}(t)\right|\right) \\
& -\xi(1+\epsilon \mu(t)) e_{\epsilon}(t, \alpha)(K+R \beta) W \\
& \times\left(\left|X_{P}\left(t-\tau_{0}\right)-X_{P}^{*}\left(t-\tau_{0}\right)\right|\right. \\
& \left.+\left|X_{N}\left(t-\tau_{0}\right)-X_{N}^{*}\left(t-\tau_{0}\right)\right|\right),
\end{aligned}
$$

which leads to

$$
\begin{aligned}
D^{+}\left(V_{1}(t)\right. & \left.+V_{2}(t)\right)\left.^{\Delta}\right|_{(1)} \\
\leq & \xi\left[\epsilon-\left(\underline{a_{P}}-R M\right)(1+\epsilon \mu(t))\right] \\
& \times e_{\epsilon}(t, \alpha)\left|X_{P}(t)-X_{P}^{*}(t)\right| \\
& +\xi\left(1+\epsilon \mu\left(t+\tau_{0}\right)\right) e_{\epsilon}\left(t+\tau_{0}, \alpha\right)(K+R \beta) W \\
& \times\left(\left|X_{P}(t)-X_{P}^{*}(t)\right|\right) \\
& +\xi\left(1+\epsilon \mu\left(t+\tau_{0}\right)\right) e_{\epsilon}\left(t+\tau_{0}, \alpha\right)(K+R \beta) W \\
& \times\left(\left|X_{N}(t)-X_{N}^{*}(t)\right|\right) .
\end{aligned}
$$

Note that

$$
\begin{aligned}
& \left.D^{+} V_{3}(t)^{\Delta}\right|_{(1)} \\
& \quad \leq \xi^{\prime}\left[\epsilon-\left(\underline{a_{N}}-R M\right)(1+\epsilon \mu(t))\right] e_{\epsilon}(t, \alpha)
\end{aligned}
$$

$$
\begin{aligned}
& \times\left|X_{N}(t)-X_{N}^{*}(t)\right|+\xi^{\prime}(1+\epsilon \mu(t)) e_{\epsilon}(t, \alpha)(K+R \beta) \\
& \times W \times\left(\left|X_{P}\left(t-\tau_{0}\right)-X_{P}^{*}\left(t-\tau_{0}\right)\right|\right. \\
& \left.\quad \quad \quad\left|X_{N}\left(t-\tau_{0}\right)-X_{N}^{*}\left(t-\tau_{0}\right)\right|\right), \\
& \left.D^{+} V_{4}(t)^{\Delta}\right|_{(1)} \\
& \leq \xi^{\prime}\left(1+\epsilon \mu\left(t+\tau_{0}\right)\right) e_{\epsilon}\left(t+\tau_{0}, \alpha\right)(K+R \beta) W \\
& \times\left(\left|X_{P}(t)-X_{P}^{*}(t)\right|+\left|X_{N}(t)-X_{N}^{*}(t)\right|\right) \\
& -\xi^{\prime}(1+\epsilon \mu(t)) e_{\epsilon}(t, \alpha)(K+R \beta) W \\
& \times\left(\left|X_{P}\left(t-\tau_{0}\right)-X_{P}^{*}\left(t-\tau_{0}\right)\right|\right. \\
& \left.\quad+\left|X_{N}\left(t-\tau_{0}\right)-X_{N}^{*}\left(t-\tau_{0}\right)\right|\right) .
\end{aligned}
$$

We have

$$
\begin{aligned}
D^{+}\left(V_{3}(t)\right. & \left.+V_{4}(t)\right)\left.^{\Delta}\right|_{(1)} \\
\leq & \xi^{\prime}\left[\epsilon-\left(\underline{a_{N}}-R M\right)(1+\epsilon \mu(t))\right] \\
& \times e_{\epsilon}(t, \alpha)\left|X_{N}(t)-X_{N}^{*}(t)\right| \\
& +\xi^{\prime}\left(1+\epsilon \mu\left(t+\tau_{0}\right)\right) e_{\epsilon}\left(t+\tau_{0}, \alpha\right)(K+R \beta) W \\
& \times\left(\left|X_{N}(t)-X_{N}^{*}(t)\right|\right)+\xi^{\prime}\left(1+\epsilon \mu\left(t+\tau_{0}\right)\right) \\
& \times e_{\epsilon}\left(t+\tau_{0}, \alpha\right)(K+R \beta) W\left(\left|X_{P}(t)-X_{P}^{*}(t)\right|\right) .
\end{aligned}
$$

From (34) and (36), we can get

$$
\begin{aligned}
D^{+} V(t)^{\Delta} & \\
\leq & \left\{\xi\left[\epsilon-\left(\underline{a_{P}}-R M\right)(1+\epsilon \mu(t))\right]\right. \\
& \left.+\left(\xi+\xi^{\prime}\right)\left(1+\epsilon \mu\left(t+\tau_{0}\right)\right) e_{\epsilon}\left(t+\tau_{0}, t\right)(K+\beta R) W\right\} \\
& \times e_{\epsilon}(t, \alpha)\left|X_{P}(t)-X_{P}^{*}(t)\right| \\
& +\left\{\xi^{\prime}\left[\epsilon-\left(\underline{a_{N}}-R M\right)(1+\epsilon \mu(t))\right]+\left(\xi+\xi^{\prime}\right)\right. \\
& \left.\times\left(1+\epsilon \mu\left(t+\tau_{0}\right)\right) e_{\epsilon}\left(t+\tau_{0}, t\right)(K+\beta R) W\right\} \\
& \times e_{\epsilon}(t, \alpha)\left|X_{N}(t)-X_{N}^{*}(t)\right| .
\end{aligned}
$$

By assumption $\left(A_{3}\right)$, it follows that $V(t) \leq V(0)$ for $t \in \mathbb{T}^{+}$. On the other hand, we have

$V(0) \leq\left[\xi e_{\epsilon}(0, \alpha)+\left(\xi+\xi^{\prime}\right)\right.$

$$
\begin{aligned}
& \left.\times \int_{-\tau_{0}}^{0}\left(1+\epsilon \mu\left(s+\tau_{0}\right)\right) e_{\epsilon}\left(s+\tau_{0}, \alpha\right)(K+\beta R) W \Delta s\right] \\
\times & \sup _{s \in\left[-\tau_{0}, 0\right]_{\top}}\left|X_{P}(s)-X_{P}^{*}(s)\right|
\end{aligned}
$$




$$
\begin{aligned}
& +\left[\xi^{\prime} e_{\epsilon}(0, \alpha)+\left(\xi+\xi^{\prime}\right)\right. \\
& \left.\quad \times \int_{-\tau_{0}}^{0}\left(1+\epsilon \mu\left(s+\tau_{0}\right)\right) e_{\epsilon}\left(s+\tau_{0}, \alpha\right)(K+\beta R) W \Delta s\right] \\
& \times \sup _{s \in\left[-\tau_{0}, 0\right]_{\mathbb{T}}}\left|X_{N}(s)-X_{N}^{*}(s)\right| \\
& =\Gamma(\epsilon)\left(\sup _{s \in\left[-\tau_{0}, 0\right]_{\mathbb{T}}}\left|X_{P}(s)-X_{P}^{*}(s)\right|\right. \\
& \left.+\sup _{s \in\left[-\tau_{0}, 0\right]_{\mathbb{T}}}\left|X_{N}(s)-X_{N}^{*}(s)\right|\right)
\end{aligned}
$$

where $\Gamma(\epsilon)=\max \left\{\Delta_{1}, \Delta_{2}\right\}$,

$$
\begin{aligned}
\Delta_{1}= & \xi e_{\epsilon}(0, \alpha)+\left(\xi+\xi^{\prime}\right) \\
& \times \int_{-\tau_{0}}^{0}\left(1+\epsilon \mu\left(s+\tau_{0}\right)\right) e_{\epsilon}\left(s+\tau_{0}, \alpha\right)(K+\beta R) W \Delta s, \\
\Delta_{2}= & \xi^{\prime} e_{\epsilon}(0, \alpha)+\left(\xi+\xi^{\prime}\right) \\
& \times \int_{-\tau_{0}}^{0}\left(1+\epsilon \mu\left(s+\tau_{0}\right)\right) e_{\epsilon}\left(s+\tau_{0}, \alpha\right)(K+\beta R) W \Delta s .
\end{aligned}
$$

It is obvious that

$$
\begin{aligned}
& \xi e_{\epsilon}(t, \alpha)\left|X_{P}(t)-X_{P}^{*}(t)\right|+\xi^{\prime} e_{\epsilon}(t, \alpha)\left|X_{N}(t)-X_{N}^{*}(t)\right| \\
& \leq V(t) \leq V(0)
\end{aligned}
$$

which means that

$$
\begin{aligned}
& \min \left\{\xi, \xi^{\prime}\right\} e_{\epsilon}(t, \alpha)\left(\left|X_{P}(t)-X_{P}^{*}(t)\right|+\left|X_{N}(t)-X_{N}^{*}(t)\right|\right) \\
& \leq V(0)
\end{aligned}
$$

Thus, we finally get

$$
\begin{aligned}
& \left|X_{P}(t)-X_{P}^{*}(t)\right|+\left|X_{N}(t)-X_{N}^{*}(t)\right| \\
& \leq \frac{\Gamma(\epsilon) e_{\ominus \epsilon}(t, \alpha)}{\min \left\{\xi, \xi^{\prime}\right\}} \times\left(\sup _{s \in\left[-\tau_{0}, 0\right]_{\mathbb{T}}}\left|X_{P}(s)-X_{P}^{*}(s)\right|\right. \\
& \left.\quad+\sup _{s \in\left[-\tau_{0}, 0\right]_{\mathbb{T}}}\left|X_{N}(s)-X_{N}^{*}(s)\right|\right) .
\end{aligned}
$$

Therefore, the unique periodic solution of (1) is globally exponentially stable. The proof is completed.

\section{Examples}

In this section, two numerical examples are shown to verify the effectiveness of the result obtained in the previous section.
Consider the following Wilson-Cowan neural network with delays on time scale $\mathbb{T}$ :

$$
\begin{aligned}
& X_{P}^{\Delta}(t) \\
& =-a_{P}(t) X_{P}(t)+\left[k_{P}(t)-r_{P}(t) X_{P}(t)\right] \\
& \quad \times G\left[w_{P}^{1}(t) X_{P}(t-2)-w_{N}^{1}(t) X_{N}(t-1)+I_{P}(t)\right], \\
& X_{N}^{\Delta}(t) \\
& =-a_{N}(t) X_{N}(t)+\left[k_{N}(t)-r_{N}(t) X_{N}(t)\right] \\
& \quad \times G\left[w_{P}^{2}(t) X_{P}(t-2)-w_{N}^{2}(t) X_{N}(t-1)+I_{N}(t)\right] .
\end{aligned}
$$

Case 1. Consider $\mathbb{T}=\mathbb{R}$. Take $\left(a_{P}(t), a_{N}(t)\right)^{\top}=(2+\sin (t), 2+$ $\cos (t))^{\top}$. Obviously, $\underline{a_{P}}=\underline{a_{N}}=1$,

$$
\begin{aligned}
& \frac{\exp \left(\int_{0}^{2 \pi} a_{P}(s) d s\right)}{\exp \left(\int_{0}^{2 \pi} a_{P}(s) d s\right)-1}=\frac{e^{4 \pi}}{e^{4 \pi}-1} \\
& \frac{\exp \left(\int_{0}^{2 \pi} a_{N}(s) d s\right)}{\exp \left(\int_{0}^{2 \pi} a_{N}(s) d s\right)-1}=\frac{e^{4 \pi}}{e^{4 \pi}-1}
\end{aligned}
$$

Take $\left(I_{P}(t), I_{N}(t)\right)^{\top}=(-1+\sin (t), \cos (t))^{\top}, k_{P}(t)=k_{N}(t)=$ $r_{P}(t)=r_{N}(t)=0.01, w_{P}^{1}(t)=w_{N}^{1}(t)=w_{P}^{2}(t)=w_{N}^{2}(t)=0.1$, and $G(x)=(1 / 2)(|x+1|-|x-1|)$. We have $L=1$. Let $\xi=1$, $\xi^{\prime}=2$. One can easily verify that

$$
\alpha_{1}=\omega\left(K+R \beta+\frac{R M}{W}\right) \frac{\exp \left(\int_{0}^{2 \pi} a_{P}(s) d s\right)}{\exp \left(\int_{0}^{2 \pi} a_{P}(s) d s\right)-1}
$$

$$
\begin{aligned}
& \approx 0.831<1, \\
& \alpha_{2}=\omega\left(K+R \beta+\frac{R M}{W}\right) \frac{\exp \left(\int_{0}^{2 \pi} a_{N}(s) d s\right)}{\exp \left(\int_{0}^{2 \pi} a_{N}(s) d s\right)-1} \\
& \approx 0.831<1, \\
& -\xi\left(\underline{a_{P}}-R M\right)+\left(\xi+\xi^{\prime}\right)(K+\beta R) W \approx-0.980<0, \\
& -\xi^{\prime}\left(\underline{a_{N}}-R M\right)+\left(\xi+\xi^{\prime}\right)(K+\beta R) W \approx-1.970<0 .
\end{aligned}
$$

It follows from Theorems 12 and 13 that (43) has a unique $2 \pi$ periodic solution which is globally exponentially stable (see Figure 1). 


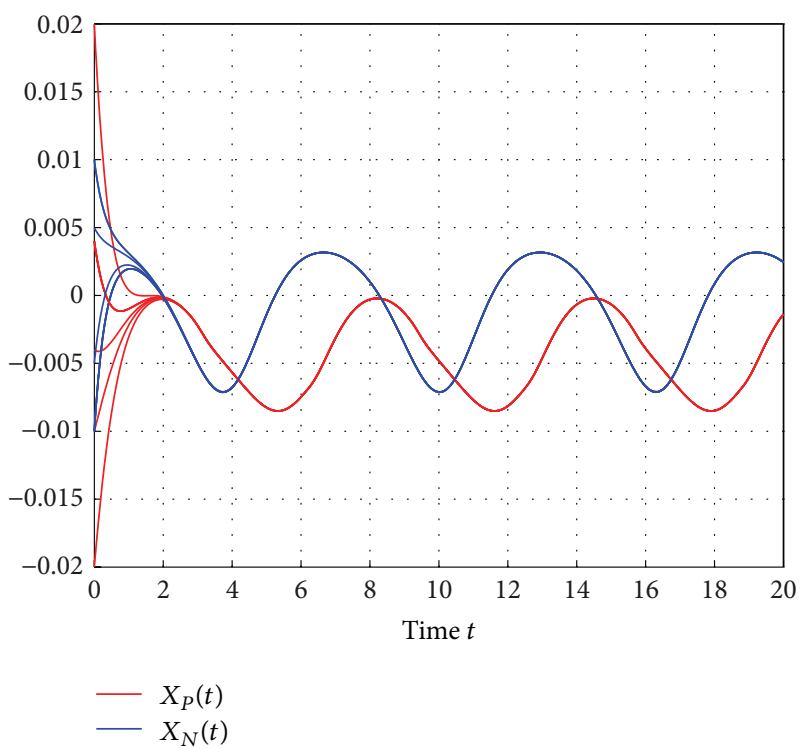

FIGURE 1: Globally exponentially stable periodic solution of (43).

Case 2. Consider $\mathbb{T}=\mathbb{Z}$. Equation (43) reduces to the following difference equation:

$$
\begin{aligned}
X_{P}(n+1)-X_{P}(n) \\
=-a_{P}(n) X_{P}(n)+\left[k_{P}(n)-r_{P}(n) X_{P}(n)\right] \\
\quad \times G\left[w_{P}^{1}(n) X_{P}(n-2)\right. \\
\left.\quad-w_{N}^{1}(n) X_{N}(n-1)+I_{P}(n)\right], \\
X_{N}(n+1)-X_{N}(n) \\
=-a_{N}(n) X_{N}(n)+\left[k_{N}(n)-r_{N}(n) X_{N}(n)\right] \\
\quad \times G\left[w_{P}^{2}(n) X_{P}(n-2)-w_{N}^{2}(n) X_{N}(n-1)+I_{N}(n)\right],
\end{aligned}
$$

for $n \in \mathbb{Z}_{0}^{+}$. Take $\left(a_{P}(n), a_{N}(n)\right)^{\top}=(1 / 2,1 / 2)^{\top}$. Obviously, $\underline{a_{P}}=\underline{a_{N}}=1 / 2, \overline{a_{P}}=\overline{a_{N}}=1 / 2,\left(I_{P}(t), I_{N}(t)\right)^{\top}=(1+$ $\overline{\sin }(n \pi / 3), \cos (n \pi / 3))^{\top}, k_{P}(t)=k_{N}(t)=r_{P}(t)=r_{N}(t)=$ $0.01, w_{P}^{1}(t)=w_{N}^{1}(t)=w_{P}^{2}(t)=w_{N}^{2}(t)=0.1$, and $G(x)=$ $(1 / 2)(|x+1|-|x-1|)$. We have $L=1$. Let $\xi=1, \xi^{\prime}=2$. If $\mathbb{T}=\mathbb{Z},(\mu(t)=1)$, choosing $\omega=6$, by simple calculation, we have

$$
\begin{aligned}
& \alpha_{1} \\
& =\left(K+R \beta+\frac{R M}{W}\right) \\
& \quad \times \frac{\omega\left|1-\prod_{k=1}^{\omega-1}\left(1-a_{P}(k)\right)\right| \exp \left(\sum_{k=0}^{\omega-1}\left|\log \left(1-a_{P}(k)\right)\right|\right)}{\left|\prod_{k=1}^{\omega-1}\left(1-a_{P}(k)\right)\right|\left(1-\overline{a_{P}}\right)} \\
& \approx 0.015<1,
\end{aligned}
$$

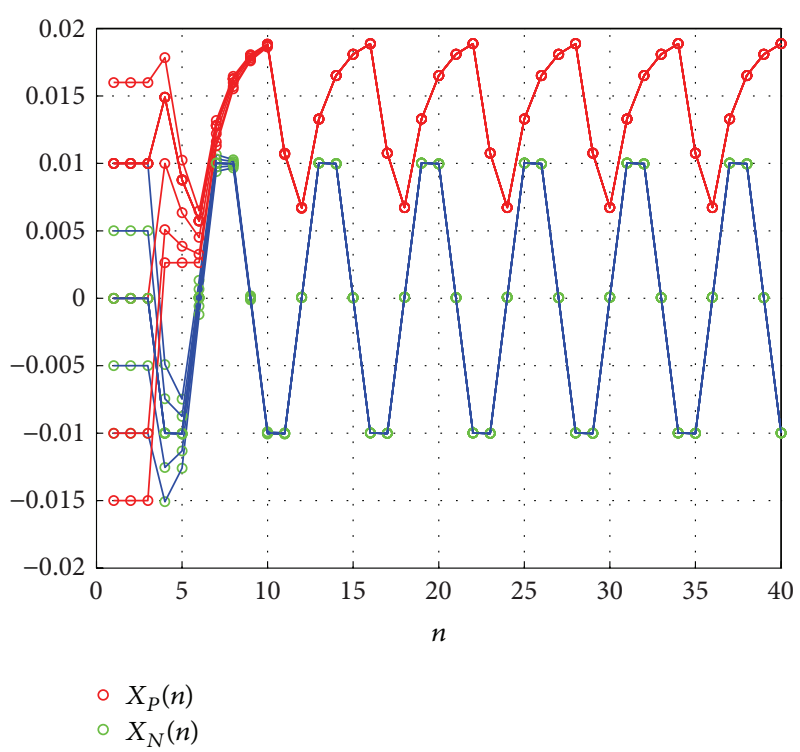

FIGURE 2: Globally exponentially stable periodic solution of (46).

$\alpha_{2}$

$$
\begin{aligned}
= & \left(K+R \beta+\frac{R M}{W}\right) \\
& \times \frac{\omega\left|1-\prod_{k=1}^{\omega-1}\left(1-a_{N}(k)\right)\right| \exp \left(\sum_{k=0}^{\omega-1}\left|\log \left(1-a_{N}(k)\right)\right|\right)}{\left|\prod_{k=1}^{\omega-1}\left(1-a_{N}(k)\right)\right|\left(1-\overline{a_{N}}\right)} \\
\approx & 0.015<1, \\
& -\xi\left(\underline{a_{P}}-R M\right)+\left(\xi+\xi^{\prime}\right)(K+\beta R) W \approx-0.480<0, \\
& -\xi^{\prime}\left(\underline{a_{N}}-R M\right)+\left(\xi+\xi^{\prime}\right)(K+\beta R) W \approx-0.970<0 .
\end{aligned}
$$

It follows from Theorems 12 and 13 that (46) has a unique 6periodic solution which is globally exponentially stable (see Figure 2).

\section{Conclusion Remarks}

In this paper, we studied the stability of delayed WilsonCowan networks on periodic time scales and obtained some more generalized results to ensure the existence, uniqueness, and global exponential stability of the periodic solution. These results can give a significant insight into the complex dynamical structure of Wilson-Cowan type model. The conditions are easily checked in practice by simple algebraic methods.

\section{Conflict of Interests}

The authors declare that there is no conflict of interests regarding the publication of this paper. 


\section{Acknowledgments}

This research was supported by the National Natural Science Foundation of China (11101187 and 11361010), the Foundation for Young Professors of Jimei University, the Excellent Youth Foundation of Fujian Province (2012J06001 and NCETFJ JA11144), and the Foundation of Fujian Higher Education (JA10184 and JA11154).

\section{References}

[1] H. R. Wilson and J. D. Cowan, "Excitatory and inhibitory interactions in localized populations of model neurons," Biophysical Journal, vol. 12, no. 1, pp. 1-24, 1972.

[2] H. R. Wilson and J. D. Cowan, "A mathematical theory of the functional dynamics of cortical and thalamic nervous tissue," Kybernetik, vol. 13, no. 2, pp. 55-80, 1973.

[3] A. Destexhe and T. J. Sejnowski, "The Wilson-Cowan model, 36 years later," Biological Cybernetics, vol. 101, no. 1, pp. 1-2, 2009.

[4] K. Mantere, J. Parkkinen, T. Jaaskelainen, and M. M. Gupta, "Wilson-Cowan neural-network model in image processing," Journal of Mathematical Imaging and Vision, vol. 2, no. 2-3, pp. 251-259, 1992.

[5] C. van Vreeswijk and H. Sompolinsky, "Chaos in neuronal networks with balanced excitatory and inhibitory activity," Science, vol. 274, no. 5293, pp. 1724-1726, 1996.

[6] L. H. A. Monteiro, M. A. Bussab, and J. G. Berlinck, "Analytical results on a Wilson-Cowan neuronal network modified model," Journal of Theoretical Biology, vol. 219, no. 1, pp. 83-91, 2002.

[7] S. Xie and Z. Huang, "Almost periodic solution for WilsonCowan type model with time-varying delays," Discrete Dynamics in Nature and Society, vol. 2013, Article ID 683091, 7 pages, 2013.

[8] V. W. Noonburg, D. Benardete, and B. Pollina, "A periodically forced Wilson-Cowan system," SIAM Journal on Applied Mathematics, vol. 63, no. 5, pp. 1585-1603, 2003.

[9] S. Hilger, "Analynis on measure chains-a unified approach to continuous and discrete calculus," Results in Mathematics, vol. 18, pp. 18-56, 1990.

[10] S. Hilger, "Differential and difference calculus-unified!," Nonlinear Analysis: Theory, Methods \& Applications, vol. 30, no. 5, pp. 2683-2694, 1997.

[11] A. Chen and F. Chen, "Periodic solution to BAM neural network with delays on time scales," Neurocomputing, vol. 73, no. 1-3, pp. 274-282, 2009.

[12] Y. Li, X. Chen, and L. Zhao, "Stability and existence of periodic solutions to delayed Cohen-Grossberg BAM neural networks with impulses on time scales," Neurocomputing, vol. 72, no. 7-9, pp. 1621-1630, 2009.

[13] Z. Huang, Y. N. Raffoul, and C. Cheng, "Scale-limited activating sets and multiperiodicity for threshold-linear networks on time scales," IEEE Transactions on Cybernetics, vol. 44, no. 4, pp. 488499, 2014.

[14] M. Bohner and A. Peterson, Dynamic Equations on Time Scales: An Introduction with Applications, Birkhäuser, Boston, Mass, USA, 2001.

[15] M. Bohner and A. Peterson, Advance in Dynamic Equations on Time Scales, Birkhäuser, Boston, Mass, USA, 2003.

[16] V. Lakshmikantham and A. S. Vatsala, "Hybrid systems on time scales," Journal of Computational and Applied Mathematics, vol. 141, no. 1-2, pp. 227-235, 2002.
[17] A. Ruffing and M. Simon, "Corresponding Banach spaces on time scales," Journal of Computational and Applied Mathematics, vol. 179, no. 1-2, pp. 313-326, 2005. 

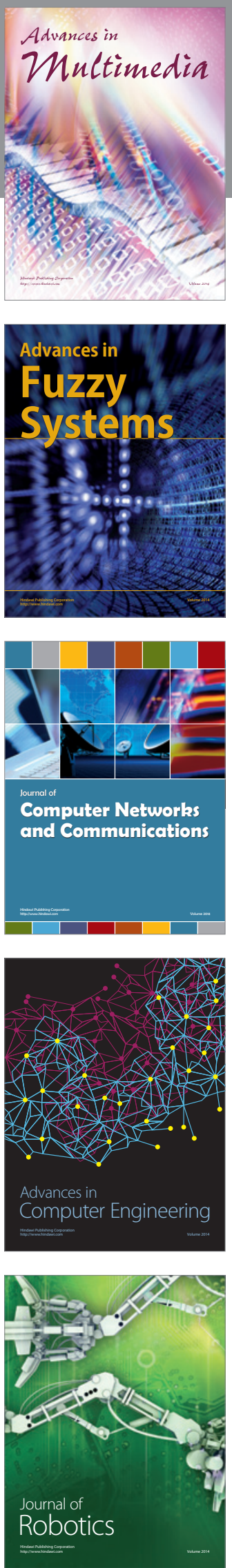

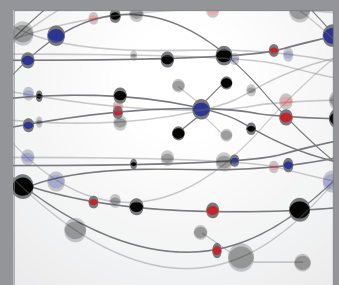

The Scientific World Journal
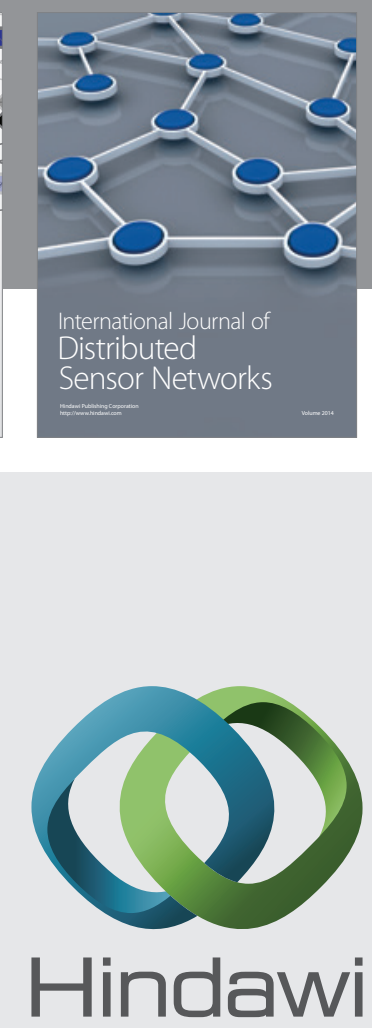

Submit your manuscripts at

http://www.hindawi.com
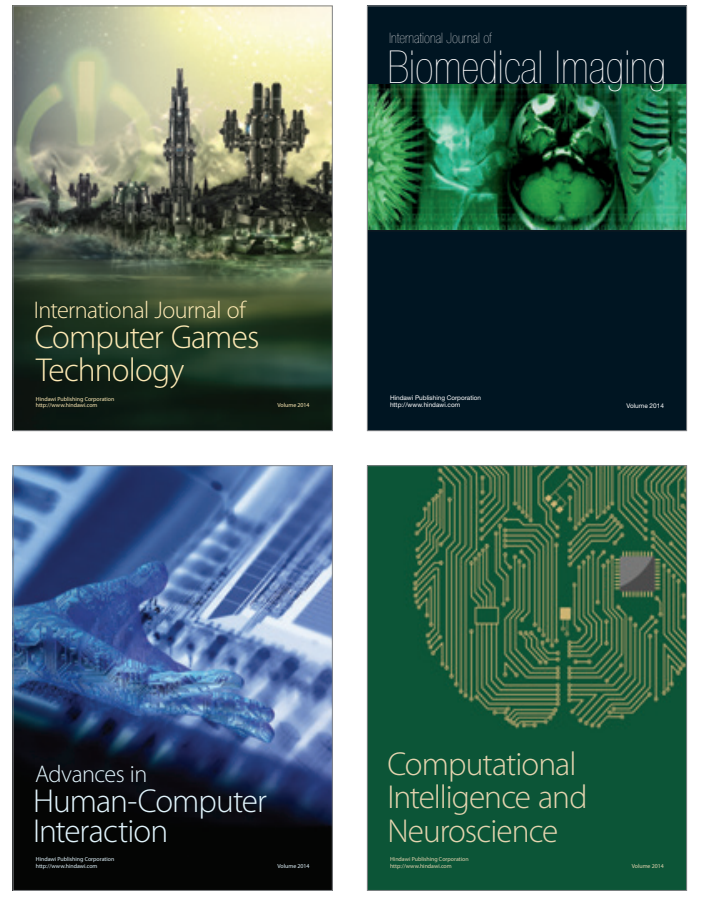
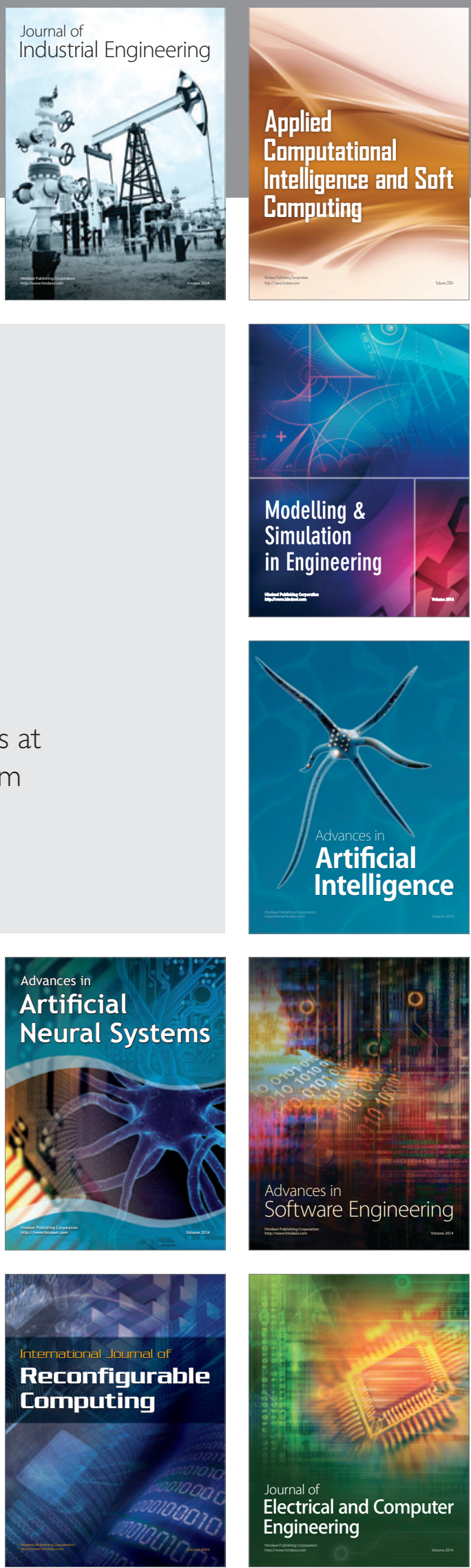